\title{
Papers
}

\section{Risk of renal stone events in primary hyperparathyroidism before and after parathyroid surgery: controlled retrospective follow up study}

\author{
Charlotte L Mollerup, Peter Vestergaard, Vibe Gedsø Frøkjær, Leif Mosekilde, Peer Christiansen, \\ Mogens Blichert-Toft
}

\begin{abstract}
Aim To study the risk of renal stone episodes and risk factors for renal stones in primary

hyperparathyroidism before and after surgery.

Design Register based, controlled retrospective follow up study.

Setting Tertiary hospitals in Denmark.

Participants 674 consecutive patients with surgically verified primary hyperparathyroidism. Each patient was compared with three age- and sex-matched controls randomly drawn from the background population. Hospital admissions for renal stone disease were compared between patients and controls. Risk factors for renal stones among patients were assessed.

Main outcome measures Number of renal stone episodes; comparison of hospital admissions for renal stones in patients and controls; assessment of risk factors for renal stones.

Results Relative risk of a stone episode was 40 (95\% confidence interval 31 to 53) before surgery and 16 (12 to 23) after surgery. Risk was increased 10 years before surgery, and became normal more than 10 years after surgery. Stone-free survival 20 years after surgery was $90.4 \%$ in patients and $98.7 \%$ in controls (risk difference $8.3 \%, 4.8 \%$ to $11.7 \%$ ). Patients with preoperative stones had 27 times the risk of postoperative stone incidents than controls. Before surgery, males had more stone episodes than females and younger patients had more stone episodes than older patients. Neither parathyroid pathology, weight of removed tissue, plasma calcium levels, nor skeletal pathology (fractures) influenced the risk of renal stones. After surgery, younger age, preoperative stones and ureteral strictures were significant risk factors for stones.

Conclusions The risk of renal stones is increased in primary hyperparathyroidism and decreases after surgery. The risk profile is normal 10 years after surgery. Preoperative stone events increase the risk of postoperative stones. Stone formers and non-stone formers had the same risk of skeletal complications.
\end{abstract}

\section{Introduction}

Primary hyperparathyroidism is associated with increased risk of renal stones. As the clinical picture of primary hyperparathyroidism has shifted towards milder and even asymptomatic cases with the advent of biochemical screening, the prevalence of stone disease has declined from around $80 \%$ in early series to $7-20 \%$ in more recent series. ${ }^{12}$

The exact pathogenesis of stone formation remains unclear. Hypercalciuria is a common finding in primary hyperparathyroidism and has been implicated in the formation of renal stones. ${ }^{13}$ The excess calcium in the urine may come from enhanced intestinal calcium absorption, increased mobilisation of skeletal calcium, or both. Increased renal production of calcitriol with hyperabsorption of intestinal calcium is believed to be clinically important. ${ }^{4-6}$

Concurrent bone and stone disease was previously considered to be rare in primary hyperparathyroidism. ${ }^{7}$ Several explanations have been proposed to account for this. Symptomatic renal stones may be diagnosed early and symptomatic severe hypercalcaemia may be diagnosed late in the natural course of the disease. Different clinical pictures may be elicited by differences in the growth potential of the parathyroid tumour; differences in dietary calcium intake; differences in the intestinal capacity for calcium absorption due to vitamin D status, age, and sex; differences in skeletal and renal responsiveness to circulating parathyroid hormone; or selective actions of unidentified factors secreted by the parathyroid gland. ${ }^{78}$ It has not been possible to document a pathogenic background for these theories. Recent studies have failed to show significant differences in intestinal calcium absorption, plasma concentrations of parathyroid hormone, and calcitriol or bone mineral density between patients with and without stone disease. ${ }^{2459}$

Several studies have investigated the course of stone disease after parathyroidectomy. ${ }^{7011}$ Parathyroidectomy reduces the risk of recurrent stone disease, provided that normocalcaemia is obtained. ${ }^{7}$ However, a $30-50 \%$ risk of recurrence after an observation period of three to five years has been reported, ${ }^{10}{ }^{11}$
Editorial by

Sørensen

Department of Endocrine and Breast Surgery Copenhagen

University Hospital, Rigshospitalet, DK 2100

Copenhagen, Denmark

Charlotte L

Mollerup associate professor Vibe Gedsø Frøkjær registrar

Mogens

Blichert-Toft professor emeritus

Department of Endocrinology and Metabolism C, Aarhus University Hospital, Aarhus Amtssygehus,

DK 8000 Aarhus, Denmark

Peter Vestergaard registrar

Leif Mosekilde professor

Department of Surgery L, Aarhus University Hospital Peer Christiansen consultant in surgery

Correspondence to: C L Mollerup molle@rh.dk

bmj.com 2002;325:807 
bringing into question the effect of parathyroidectomy in a substantial proportion of the patients.

In our retrospective follow up study, which included 674 patients and 2021 population based controls, we investigated several research questions: is the risk of renal stones increased in patients with primary hyperparathyroidism before surgery compared with the background population; to what extent do patients with and without kidney stones differ in variables such as age, sex, severity of disease, histopathology, and skeletal symptoms; and whether surgery decreases the future risk of stone disease.

\section{Methods}

The study was designed as a register based, controlled retrospective follow up study.

\section{Patients}

The cohort consisted of 674 consecutive white patients with surgically verified primary hyperparathyroidism treated at Aarhus University Hospital between 1979 and 1997, at Odense University Hospital between 1979 and 1990, and at Copenhagen University Hospital, Rigshospitalet, between 1991 and 1997. History of stones before operation and biochemical and histopathological data were retrieved from the medical files. Of the 674 patients, $97 \%$ (653) were operated on within one year after the diagnosis was first registered. All patients included were normocalcaemic after surgery.

\section{Controls}

For each of the 674 patients, three white controls frequency matched for age (birth year), sex, and status were drawn from population lists of people residing in Denmark between 1 January 1979 and 31 December 1997. The status match regarded death or emigration. If the case had died or emigrated, a control who had died or emigrated on the same date or later than the case was chosen. The Danish Ministry of the Interior performed this matching by standard database queries involving the national person register as previously described. ${ }^{12}$ One case (dead) could be matched with only two controls, leaving 2021 controls to be matched with the 674 patients. Each control was assigned a "dummy date of diagnosis"- the date on which the corresponding case's primary hyperparathyroidism was diagnosed. The follow up period of the controls was then divided into the period before and after this date. $^{12}$

Table 1 Clinical characteristics and operative findings in 674 patients with primary hyperparathyroidism. Values are mean (SD) unless otherwise stated

\begin{tabular}{lccc} 
Characteristic & All patients $(\mathbf{n}=\mathbf{6 7 4})$ & Men $(\mathbf{n}=\mathbf{1 7 3})$ & Women $(\mathbf{n}=\mathbf{5 0 1})$ \\
\hline Age (years) & $58.2(15.0)$ & $54.8(16.6)$ & $59.4(14.3)$ \\
\hline No (\%) with adenomas & $606(90)$ & $155(90)$ & $451(90)$ \\
\hline No (\%) with hyperplasia & $68(10)$ & $18(10)$ & $50(10)$ \\
\hline Adenoma weight $(\mathrm{g})$ & $2.11(5.18)$ & $3.03(8.79)$ & $1.80(3.13)$ \\
\hline Median (range) & $1.00(0.02-87)$ & $1.20(0.03-87)$ & $1.00(0.02-40)$ \\
\hline Hyperplasia weight $(\mathrm{g})$ & $3.59(6.53)$ & $3.57(4.29)$ & $3.60(7.09)$ \\
\hline Median (range) & $1.50(0.10-42)$ & $2.82(0.80-17.61)$ & $1.48(0.10-42)$ \\
\hline Stone disease mentioned in file & $167(25)$ & $58(34)$ & $109(22)$ \\
\hline Serum calcium (ionised), mmol//* & $1.58(0.17)$ & $1.59(0.15)$ & $1.57(0.17)$ \\
\hline Postoperative observation period (years) & $5.4(4.4)$ & $6.9(4.4)$ & $5.3(4.3)$ \\
\hline${ }^{*}$ Measured on day before surgery. & & &
\end{tabular}

\section{Outcome variables}

For both patients and controls, all inpatient and outpatient discharge diagnoses concerning kidney or urinary tract stones (ICD-8 codes 592.00 (renal stone(s)), 592.01 (multiple renal stones), 592.02 (coral stone), 592.03 (ureter stone(s)), 592.04 (pyelonephritis with stone(s)), 592.05 (pyonephrosis with stone(s)), 592.08 (other types of stones in pelvis or ureter), 592.09 (stones in pelvis and ureter); ICD-10 codes N20.0 (renal stone(s)), N20.1 (ureter stone(s)), N20.2 (stones in pelvis and ureter), N20.9 (renal stones without specification)) between 1 January 1978 and 31 December 1997 were retrieved from the National Patient Register under the Danish National Board of Health. ${ }^{13}$ Only discharges in which renal stone disease was the main diagnosis were considered for statistical evaluation.

Furthermore, for both patients and controls, information on any fracture diagnosis (ICD-8 codes 800.00813.99, 820.00-824.99; ICD-10 codes S02.0-S02.9, S07.0-S07.9, S12.0-S12.9, S22.0-S22.9, S32.0-S32.9, S42.0-S42.9, S52.0-S52.9, S72.0-S72.9, S82.0-S82.9) or osteoporosis (ICD-8 code 723.09; ICD-10 codes M80.0-M81.9) was retrieved from the same source.

Serum calcium was measured as ionised serum calcium on an automated ionised calcium analyser using an ion selective electrode (Radiometer, Copenhagen) in patients who had surgery at Copenhagen University Hospital. In Odense and Aarhus serum calcium was measured as albumin adjusted total serum calcium on a multichannel autoanalyser in the daily hospital routine; these values were adjusted to ionised calcium by the formula:

ionised serum calcium $(\mathrm{mmol} / \mathrm{l})=0.25+0.45 \times$ serum total calcium $(\mathrm{mmol} / \mathrm{l})$. All calcium values reported were measured the day before surgery.

\section{Statistics}

Mean, standard deviation, median, and range were applied as descriptive statistics. The Mann-Whitney test or the $\chi^{2}$ test for contingency tables were used to compare numbers where appropriate. Incidence rates were calculated as the number of participants with at least one stone-related admission divided by the observation time and were compared as incidence rate ratios by Mantel-Haenszel-type statistics. Differences between proportions were calculated by Fischer's exact test. Incidence rate ratios were compared by using Poisson regression. Risk factors were analysed by using logistic regression and Cox regression. Stone-free survival was determined from Kaplan-Meier plots. SPSS 6.1.3 for Windows was used for all calculations.

\section{Results}

Table 1 shows details of the 674 patients. Women made up $74 \%$ of the cohort, of whom $90 \%$ (451) had parathyroid adenoma and 10\% (50) had hyperplasia. The amount of removed tissue varied greatly. Male patients were younger and had larger adenomas and a higher incidence of stone disease than female patients.

Risk of stone disease and effect of parathyroid surgery

The incidence rate ratio of contact with hospital because of stone disease was 40.6 (95\% confidence interval 31.0 to 53.3) before parathyroid surgery and 
Table 2 Incidence rate ratio of renal stone event in patients and controls before and after surgery

\begin{tabular}{|c|c|c|c|c|c|c|c|}
\hline \multirow[b]{3}{*}{ Group } & \multicolumn{3}{|c|}{ Before surgery } & \multicolumn{3}{|c|}{ After surgery } & \multirow[b]{3}{*}{$P$ value* } \\
\hline & \multicolumn{2}{|c|}{ Events/years } & \multirow{2}{*}{$\begin{array}{c}\text { Incidence rate ratio } \\
(95 \% \mathrm{Cl})\end{array}$} & \multicolumn{2}{|c|}{ Events/years } & \multirow{2}{*}{$\begin{array}{l}\text { Incidence rate ratio } \\
(95 \% \mathrm{Cl})\end{array}$} & \\
\hline & Patients & Controls & & Patients & Controls & & \\
\hline All patients & $271 / 9229$ & $20 / 27670$ & 40.6 (31.0 to 53.3$)$ & $112 / 3661$ & $22 / 12038$ & 16.9 (12.1 to 23.6$)$ & $<0.01$ \\
\hline $\begin{array}{l}\text { Patients with stones before } \\
\text { operation }\end{array}$ & - & - & - & $97 / 1111$ & $4 / 3476$ & 75.9 (45.8 to 125.5$)$ & - \\
\hline $\begin{array}{l}\text { Patients without stones } \\
\text { before operation }\end{array}$ & - & - & - & $15 / 2517$ & $18 / 8550$ & 2.8 (1.5 to 5.5$)$ & - \\
\hline $\begin{array}{l}\text { Patients with strictures of } \\
\text { ureters or hydronephrosis }\end{array}$ & $3 / 9229$ & $27 / 27670$ & 9.0 (1.4 to 58.1$)$ & $9 / 3631$ & $1 / 12038$ & 29.8 (7.9 to 112.7$)$ & 0.30 \\
\hline
\end{tabular}

16.9 (12.1 to 23.6) after surgery $(\mathrm{P}<0.01$, table 2$)$. Figure 1 shows a time resolved curve of the relative risk of stone disease. The risk was highest within the four years before and in the four years after surgery, with a peak in risk immediately before diagnosis and treatment. The risk of a stone event was significantly increased as much as 10 years before the diagnosis of primary hyperparathyroidism. From five to 10 years after surgery the risk of stone events remained at the same level as five to 10 years before surgery. However, more than 10 years after surgery the risk of stone events in patients did not differ from the risk among controls.

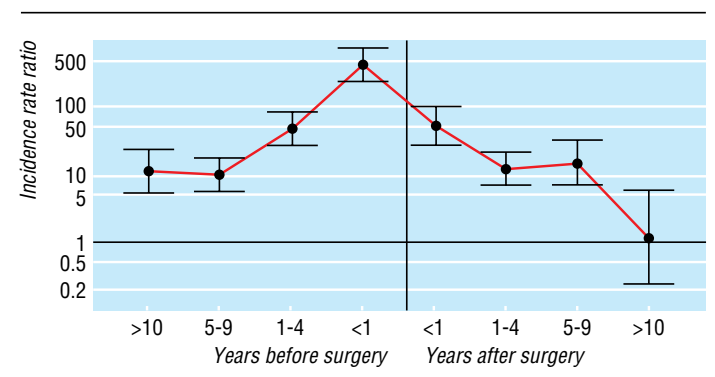

$\begin{array}{lcccccccc}\text { Events: } & & & & & & & & \\ \text { Patients } & 20 & 31 & 81 & 139 & 54 & 37 & 19 & 2 \\ \text { Controls } & 5 & 9 & 5 & 1 & 3 & 9 & 4 & 6 \\ \text { Person years: } & & & & & & & & \\ \text { Patients } & 3049 & 2901 & 2604 & 674 & 619 & 1727 & 946 & 339 \\ \text { Controls } & 9141 & 8699 & 7809 & 2021 & 1890 & 5551 & 3275 & 1321\end{array}$

Fig 1 Renal stone events among patients compared with controls before and after parathyroid surgery. Bars indicate $95 \%$ confidence intervals; vertical line indicates time of surgery

Figure 2 shows renal stone-free survival after surgery in patients and their controls. The stone-free survival after surgery was $98.7 \%$ in controls and $90.4 \%$ in patients 20 years after surgery, yielding a risk difference of $8.3 \%(4.8-11.7 \%)$.

Patients with preoperative stones had 27 times the risk of postoperative stone incidents (incidence rate ratio 75.9) of patients without preoperative stones (incidence rate ratio 2.8; table 2).

The risk of strictures of the ureters and hydronephrosis was higher in patients than in controls both before and after parathyroid surgery. All three patients with ureteral stricture or hydronephrosis before diagnosis also had urinary tract stones; one of the three patients diagnosed as having postoperative strictures or hydronephrosis did not have concomitant urinary tract stones.

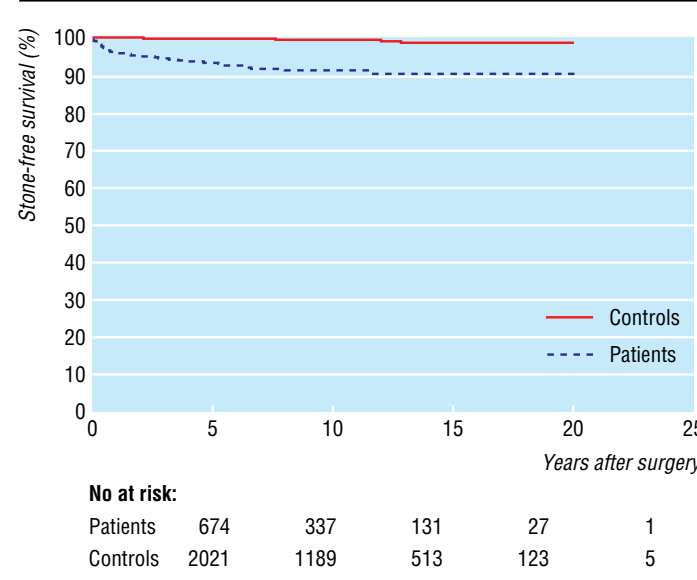

Fig 2 Stone-free survival after surgery

\section{Differences between stone formers and non-stone} formers

Apart from a significantly lower age at diagnosis and a higher proportion of men among patients with stones, no significant differences were evident in patients with and without renal stones (table 3 ). The groups did not differ in parathyroid pathology, weight of removed tissue, or plasma calcium concentrations. Skeletal complications in terms of fractures or a diagnosis of osteoporosis were similar in the two groups.

\section{Risk factors for renal stones}

Before surgery, men had more stone episodes than women, and younger patients had more stone episodes

Table 3 Characteristics of patients with or without preoperative renal stone episodes. Values are means (SD)

\begin{tabular}{lccr} 
Variable & $\begin{array}{c}\text { Patients with } \\
\text { stones }(\mathbf{n}=\mathbf{1 6 7})\end{array}$ & $\begin{array}{c}\text { Patients without } \\
\text { stones }(\mathbf{n}=\mathbf{5 0 6})\end{array}$ & P value \\
\hline Age (years) & $53.0(15.5)$ & $59.8(14.5)$ & $<0.01^{*}$ \\
\hline Weight of diseased tissue $(\mathrm{g})$ & $1.95(3.99)$ & $2.36(5.72)$ & $0.42^{*}$ \\
\hline Median (range) & $1.19(0.02-40)$ & $1.01(0.03-87)$ & \\
\hline No (\%) of patients with adenoma & $457(90)$ & $148(89)$ & $0.53 \dagger$ \\
\hline No (\%) of patients with hyperplasia & $49(10)$ & $19(11)$ & \\
\hline Serum ionised calcium (mmol//)§ & $1.57(0.14)$ & $1.58(0.17)$ & $0.72^{*}$ \\
\hline Male:female ratio & $35: 65$ & $23: 77$ & $<0.01 \dagger$ \\
\hline No (\%) of patients with osteoporosis before & $1(1)$ & $14(3)$ & $0.13 \ddagger$ \\
diagnosis & & & \\
\hline No (\%) of patients with osteoporosis after diagnosis & 0 & $7(1)$ & $0.20 \ddagger$ \\
\hline No (\%) of patients with fracture before diagnosis & $17(10)$ & $57(11)$ & $0.70 \dagger$ \\
\hline No (\%) of patients with fracture after diagnosis & $11(7)$ & $47(9)$ & $0.28 \dagger$ \\
\hline
\end{tabular}

Data on stones before operation were missing in one patient.

*Independent samples $t$ test (two sided).

$\dagger \chi^{2}$ test.

†Fisher's exact test (two sided).

$\S$ Measured on the day before surgery. 
Table 4 Risk factors for kidney stones and urinary tract stones among the patients. Values are risk estimates ( $95 \%$ confidence intervals)

\begin{tabular}{|c|c|c|c|c|}
\hline \multirow[b]{2}{*}{ Variable } & \multicolumn{2}{|c|}{ Unadjusted odds ratio } & \multicolumn{2}{|c|}{ Adjusted risk estimate } \\
\hline & Before surgery & After surgery & Before surgery† & After surgerył \\
\hline Age $(\geqslant 60 v<60$ years $)$ & $0.34(0.22 \text { to } 0.52)^{*}$ & $0.28(0.15 \text { to } 0.54)^{\star}$ & $0.33(0.20 \text { to } 0.54)^{*}$ & $0.44(0.20 \text { to } 0.96)^{\star}$ \\
\hline Preoperative stones (yes/no) & - & $11.13(6.50 \text { to } 19.08)^{*}$ & - & $5.89(3.04 \text { to } 11.41)^{*}$ \\
\hline Weight of diseased tissue $(\geqslant 1 \mathrm{~g} v<1 \mathrm{~g})$ & 1.05 (0.67 to 1.64$)$ & $1.05(0.56$ to 1.98$)$ & $0.91(0.56$ to 1.48$)$ & $1.03(0.54$ to 1.96$)$ \\
\hline Sex (male/female) & $2.02(1.31 \text { to } 3.11)^{\star}$ & $2.63(1.46 \text { to } 4.75)^{\star}$ & $1.83(1.11 \text { to } 3.00)^{*}$ & 1.51 (0.79 to 2.92$)$ \\
\hline Serum ionised calcium $(>1.55 \mathrm{mmol} / \mathrm{l} v \leqslant 1.55 \mathrm{mmol} / \mathrm{l}) \S$ & $0.91(0.59$ to 1.40$)$ & $0.97(0.52$ to 1.81$)$ & $1.09(0.67$ to 1.78$)$ & $0.86(0.45$ to 1.65$)$ \\
\hline
\end{tabular}

${ }^{*} \mathrm{P}<0.05$.

†Logistic regression (odds ratio).

‡Cox regression (hazard ratio).

$\S$ Measured the day before surgery.

than older patients (table 4 ). Neither the weight of removed parathyroid tissue nor preoperative plasma calcium was related to the risk estimate. Also, after surgery younger patients had more stone episodes than older patients. Furthermore, a history of stones was associated with more stone episodes after surgery.

\section{Discussion}

Patients with primary hyperparathyroidism had a greater risk of renal stone disease even 10 years before the diagnosis was registered than did a population based control group matched for sex and age. This is in accordance with the higher risk of fractures observed in the same population 10 years before diagnosis. ${ }^{12}$ These findings suggest that the disease has started several years before diagnosis and emphasises the importance of early diagnosis and treatment. This view is further supported by the finding of a higher risk of stone events before diagnosis. Parathyroid adenomas were larger and preoperative serum calcium concentrations were higher in our patients than in other surgical series, ${ }^{14}{ }^{15}$ supporting the theory of a delay in diagnosis.

However, other explanations of our findings regarding the delay in diagnosis of renal stone disease may exist. Because plasma calcium concentrations are routinely determined in Danish patients with renal stones, our findings may resurrect the possibility of symptomatic normocalcaemic hyperparathyroidism, an entity widely debated in the $1970 \mathrm{~s}^{7}{ }^{76}$ Another possibility is that idiopathic renal hypercalciuria may, in some cases, be the cause of later primary hyperparathyroidism. ${ }^{5}$ This would explain why renal stone disease occurs many years before diagnosis, and it agrees with the persistent increase in the risk of renal stones several years after parathyroid surgery, especially among patients who previously had stones.. This interpretation presumes that the primary renal calcium leak continues after parathyroidectomy. Our findings of an increased risk of fractures up to 10 years before diagnosis ${ }^{12}$ does not contradict this theory because renal calcium loss may cause increased bone turnover and bone loss, leading to decreased skeletal integrity.

\section{Relation between diseases}

Our data do not unequivocally indicate a causal relation between primary hyperparathyroidism and renal stone disease. In evaluating a relation between two common diseases such as renal stone disease and primary hyperparathyroidism, the possibility of confounding by indication should be considered. The diagnosis of primary hyperparathyroidism depends on plasma calcium concentration. The risk of being diagnosed with a diagnosis of primary hyperparathyroidism would be more likely in patients who had a renal stone event than in controls, who are not submitted to systematic plasma calcium screening (Berkson's bias). The peak in hospital admissions for renal stones around the time of diagnosis corroborates this interpretation.

The epidemiological observation of an increased risk of renal stone disease many years before any clinical suspicion of primary hyperparathyroidism also indicates that biological associations exist between primary hyperparathyroidism and renal stone disease. The fact that a substantial proportion of patients never experience renal stones, and the observation that the risk of stone formation is decreasing with the growing awareness of the disease, ${ }^{2} 17$ suggest that there are factors that modulate the biological relation between primary hyperparathyroidism and renal stone disease.

Our study did not corroborate the previous finding that patients with nephrolithiasis had smaller tumour weight and lower serum calcium concentrations than patients with skeletal disorders. ${ }^{78}$ In the study by Lloyd, the specific hyperparathyroid disorder of osteitis fibrosa was used as an indicator of skeletal disease, ${ }^{18}$ whereas we used the occurrence of skeletal fractures as an indicator.

\section{Surgery and risk}

After surgical treatment of primary hyperparathyroidism, hospital admissions due to stone episodes were reduced. Without randomisation to surgery or no surgery, one cannot determine whether this reduction in stone episodes is caused by the treatment, by the natural course of the disease, or by the synchronisation effect induced by a possible Berkson's bias. In our study, the risk of new renal stone episodes before, as well as after, parathyroid surgery was lower with older age. However, the reduction of stone events after diagnosis and surgery was significantly higher than could be explained by the average increase in age during follow up, suggesting a treatment effect of around $8 \%$. The risk of hospital admission because of stone disease returned to normal more than 10 years after surgery (fig 1).

Patients also had a higher risk of strictures of the ureters and of hydronephrosis than the controls both before and after surgery. This could indicate that stones caused anatomical damage to the urinary tract. These structural changes could increase the risk of having stones subsequently even though the biochemical abnormalities had been normalised by surgery.

Our data accord with recent studies that have shown that patients with primary hyperparathyroidism 


\section{What is already known on this topic}

Patients with primary hyperparathyroidism have an increased risk of renal stone events

The extent to which parathyroid surgery reduces the risk of further stones is unclear

\section{What this study adds}

The risk of a new stone event was $8.3 \%$ higher in patients than in controls after surgery

In patients with stone disease before operation the risk rate for a postoperative stone event was 27times that in controls

The risk of a renal stone event was higher than the risk among controls until more than 10 years after surgery

and renal stones may continue to produce stones after parathyroid surgery even though normocalcaemia has been established. ${ }^{10} 11$ Patients with renal stones who have primary hyperparathyroidism have a higher renal calcium excretion than those without stones who have similar concentrations of plasma calcium. ${ }^{19}$ Finally, three years after parathyroidectomy, patients who continue to form stones have higher average renal calcium excretion than those without stones. This suggests again that idiopathic renal hypercalciuria could have a pathogenic role in patients with recurrent stone formation. $^{20}$

\section{Conclusions}

Primary hyperparathyroidism is associated with an increased risk of renal stones more than 10 years before the diagnosis is established. Parathyroidectomy is associated with an $8.3 \%$ risk reduction in renal stone events, and more than 10 years after surgery the risk returns to that of controls. Male sex, younger age, and previous stone events are associated with a greater risk of stone disease. The study does not support the concept of dividing primary hyperparathyroidism patients into separate clinical entities of patients who form stones and patients with skeletal complications.

Contributors: CLM drafted the paper and PV made the statistical preparation of the data. CLM, PC, and MBT operated on the patients. VGF coordinated the data collection. LM did the clinical control of the patients. All authors participated in data collection, interpretation of data, and revision of the manuscript. CLM and PV are guarantors.

Funding: None.

Competing interests: None declared.

1 Klugman VA, Favus MJ, Pak CYC. Nephrolithiasis in primary hyperparathyroidism. In: Bilezikian JP, Marcus R, Levine MA, eds. The parathyroids: basic and clinical concepts. New York: Raven, 1994:505-17.

2 Silverberg SJ, Shane E, Jacobs TP, Siris ES, Gartenberg F, Seldin D, et al. Nefrolithiasis and bone involvement in primary hyperparathyroidism. Am J Med 1990;89:327-34

3 Coe LF, Parks JL, Asplin JR: The pathogenesis and treatment of kidney stones. N Engl J Med 1992;327:1141-52.

4 Mosekilde L, Charles P, Lindegreen P. Determinants for serum 1,25-dihydroxycholecalciferol in primary hyperparathyroidism. Bone Miner 1989;5:279-90.

5 D'Angelo A, Lodetti MG, Giannini S, Castrignano R, alAwasy M, Malvas $\mathrm{L}$, et al. Hyperparathyroidism: cause or consequence of recurrent calcium nefrolithiasis. Miner Electrolyte Metab 1992;18:359-64.

6 Broadus AE, Horst RL, Lang R, Littledike ET, Rasmussen H. The importance of circulating 1,25-dihydroxyvitamin $\mathrm{D}$ in the pathogenesis of hypercalciuria and renal stone formation in primary hyperparathyroidism. N Engl J Med 1980;302:421-6.

7 Halabe A, Sutton AL. Primary hyperparathyroidism as a cause of calcium nefolithiasis. In: Coe FL, Murray JF, eds. Disorders of bone and mineral metabolism. New York: Raven, 1992:671-84.

8 Parfitt M. Parathyroid growth: normal and abnormal. In: Bilezikian JP, Marcus R, Levine MA, eds. The parathyroids: basic and clinical concepts. New York: Raven, 1994:373-405.

9 Pak CYC, Nicar MJ, Peterson R, Zerwekh JE, Snyder W. A lack of unique pathophysiologic background for nephrolithiasis of primary hyperparathyroidism.J Clin Endocrinol Metab 1981;53:536-42.

10 Mollerup CL, Lindewald $\mathrm{H}$. The natural history of renal stone disease after successful parathyroidectomy. World J Surg 1999;23:173-6.

11 Posen S, Clifton-Bligh P, Reeve TS, Wagstaffe C, Wilkinson M. Is parathyroidectomy of benefit in primary hyperparathyroidism? QJ Med 1985; 241-51.

12 Vestergaard P, Mollerup CL, Frokjaer V, Christiansen P, Blichert-Toft M, Mosekilde L. Cohort study of risk of fracture before and after surgery for primary hyperparathyroidism. BMJ 2000;321:598-602.

13 Andersen TF, Madsen M, Jorgensen J, Mellemkjaer L, Olsen JH: The Danish national hospital register. Dan Med Bull 1999;46:263-8.

14 Williams JG, Wheeler MH, Aston JP, Brown RC, Woodhead JS. The relationship between adenoma weight and intact (1-84) parathyroid hormone level in primary hyperparathyroidism. Am $J$ Surg 1992;163:301-4.

15 Hedback G, Oden A, Tisell LE. Parathyroid adenoma weight and the risk of death after treatment for primary hyperparathyroidism. Surgery $1995 ; 117: 134-9$.

16 Johansson H, Thorén L, Werner I, Grimelius L. Normocalcemic hyperparathyroidism, kidney stones and idiopatic hypercalciuria. Surgery 1975;77:691-6.

17 Åkerstrøm G. Non-familial hyperparathyroidism. Semin Surg Onco 1997;13:104-13.

18 Lloyd HM. Primary hyperparathyroidism. An analysis of the role of the parathyroid tumor. Medicine 1968;47:53-71.

19 Söreide JA, Heerden JA, Grant CS, Lo CY, Ilstrup DM. Characteristics of patients surgically treated for primary hyperparathyroidism with and without renal stones. Surgery 1996;120:1033-7.

20 Froekjaer V, Mollerup CL. Primary hyperparathyroidism: renal calcium excretion in patients with and without renal stones before and after parathyroidectomy. World J Surg 2002;26:532-35.

(Accepted 21 May 2002) 